\title{
ASPECTOS FLORÍSTICOS E ECOLÓGICOS DE EPÍFITOS VASCULARES SOBRE FIGUEIRAS ISOLADAS NO NORTE DA PLANÍCIE COSTEIRA DO RIO GRANDE DO SUL
}

\author{
Cezar Neubert Gonçalves ${ }^{1}$ \\ Jorge Luiz Waechter ${ }^{2}$
}

Recebido em 11/01/2001. Aceito em 17/05/2002

\begin{abstract}
RESUMO - (Aspectos florísticos e ecológicos de epífitos vasculares sobre figueiras isoladas no norte da planície costeira do Rio Grande do Sul). Estudos com epífitos vasculares no Brasil normalmente não consideram árvores isoladas em áreas antropizadas, as quais permitem o estabelecimento e preservação de uma porção representativa da flora epifítica original. Neste trabalho, enfoca-se a composição florística dos epífitos vasculares em espécimes isolados de Ficus organensis (Miq.) Miq. no norte da planície costeira do Rio Grande do Sul. A área de estudo fica situada no entorno da cidade de Terra de Areia (29 $35^{\prime}$ 'S e $\left.50^{\circ} 04^{\prime} \mathrm{W}\right)$, com clima subtropical úmido (Cfa). Sessenta árvores foram inventariadas. Foram encontradas 77 espécies, 33 gêneros e 10 famílias. A família Orchidaceae e o gênero Tillandsia L. apresentaram os maiores números de espécies. A categoria ecológica mais diversificada foi a dos holoepífitos, com 69 espécies, a maioria delas apresentando a suculência como adaptação para o estresse hídrico. A proporção de espécies anemocóricas (51) foi praticamente o dobro das zoocóricas (26). A percentagem das espécies epifíticas, em relação à flora epifítica regional, foi de $30,8 \%$. A proporção de Orchidaceae foi relativamente menor, provavelmente devido a maiores exigências em relação aos hábitats florestais originais. A família com maior importância fisionômica foi Bromeliaceae. O predomínio das espécies anemocóricas sobre as zoocóricas, nas árvores amostradas, é menor do que em áreas com florestas preservadas.
\end{abstract}

Palavras-chave - epifitismo, florística, ecologia, Ficus organensis, Sul do Brasil

ABSTRACT - (Floristic and ecological aspects of vascular epiphytes on isolated fig trees on the northern coastal plain of Rio Grande do Sul). Studies on vascular epiphytes in Brazil usually do not consider isolated trees close to anthropic areas. These trees allow the establishment and preservation of a representative proportion of the original epiphytic flora. In this study, the floristic composition of vascular epiphytes was surveyed on isolated specimens of Ficus organensis (Miq.) Miq., on the northern coastal plain of Rio Grande do Sul. The study area lies around the city of Terra de Areia ( $29^{\circ} 35^{\prime} \mathrm{S} ; 5^{\circ} 04^{\prime} \mathrm{W}$ ), a region with a humid subtropical climate (Cfa). Sixty trees were surveyed. The floristic survey resulted in 77 species, 33 genera and 10 families. Orchidaceae and the genus Tillandsia L. presented the highest number of species. The most diversified ecological category was true epiphytes, with 69 species, most of these are succulent as an adaptation to water deficit. The proportion of

1 Doutorando em Botânica - UFRGS/CAPES, Bolsista do CNPq no período 1998-2000

2 Professor do PPG Botânica/UFRGS. Av. Bento Gonçalves, 9500, Bloco 4, Prédio 43433, 91570-900, Porto Alegre, RS, Brasil(jorgew@myway.com.br) 
anemochoric species (51) was almost twice the number of zoochoric species (26). The percentage of epiphytic species in relation to the regional epiphytic flora was 30.8\%. The proportion of Orchidaceae was relatively small, probably due to greater ecological demands in relation to the original forested habitats. The family with greatest physiognomic importance was Bromeliaceae. Although anemochoric species clearly predominate over zoochoric species, the importance of this seed dispersal form is still higher in areas with well-preserved forests.

Key words - epiphytism, floristics, ecology, Ficus organensis, Southern Brazil

\section{Introdução}

Os epífitos constituem um contingente importante da flora vascular, especialmente em regiões tropicais e subtropicais úmidas (Benzing 1995; Coxson \& Nadkarni 1995; Andrade \& Nobel 1997; Dislich \& Mantovani 1998; Guevara et al. 1998; Waechter 1998a). Sua composição taxonômica tem sido abordada em levantamentos amplos, incluindo toda a flora vascular (Kelly et al. 1994; Roldán 1995; Galeano et al. 1998; Moguel \& Toledo 1999), ou específicos desta sinúsia (Braga \& Braga 1975; Aguiar et al. 1981; Waechter 1986, 1998b; Brown 1990; Gottsberger \& Morawetz 1993; Wolf 1994; Hietz \& Hietz-Seifert 1995; Jarman \& Kantvilas 1995; Atzingen et al. 1996; Ingram et al. 1996; Fontoura et al. 1997; Labiak \& Prado 1998; Dittrich et al. 1999; Nieder et al. 1999; Silva et al. 1999; Kersten \& Silva 2001). Por outro lado, a realização de estudos sobre a composição de epífitos vasculares que ocorrem em monoculturas arbóreas, em áreas tropicais, ou em pteridófitas arborescentes, em ilhas oceânicas, têm fornecido um modelo simplificado para pesquisar aspectos florísticos e fitossociológicos, que normalmente são difíceis de serem abordados em ambientes florestais (Yeaton \& Gladstone 1982; Catling et al. 1986; Heatwole 1990; Medeiros et al. 1993; Nadarajah \& Nawawi 1993). A atenção dos pesquisadores também tem se voltado para as árvores remanescentes, que ficam isoladas entre plantações, pastagens ou mesmo em áreas urbanas, sobre as quais a flora epifítica pode se desenvolver e ter parte significativa de sua biodiversidade preservada (Willians-Linera et al. 1995; Guevara et al. 1998).
Orchidaceae e Bromeliaceae constituem, normalmente, as famílias dominantes na região neotropical e, juntamente com Cactaceae e Polypodiaceae, costumam destacar-se na fisionomia do componente epifítico (Ribeiro et al. 1994; Benzing 1995; Hietz 1997; Nunes \& Waechter 1998; Waechter 1998a). No Brasil, os trabalhos enfocando o epifítismo vascular são poucos e foram realizados em áreas florestais (Waechter 1986, 1998b; Fontoura et al. 1997; Dislich \& Mantovani 1998; Nunes \& Waechter 1998; Dittrich et al. 1999; Kersten \& Silva 2001) ou savanas (Braga \& Braga 1975; Gottsberger \& Morawetz 1993). Em todos estes trabalhos, o padrão florístico encontrado é semelhante, com predomínio das famílias referidas anteriormente e de holoepífitos habituais. Nenhum deles, porém, abordou as árvores que ocorrem isoladas em áreas antropizadas, não havendo, para o Brasil, referência alguma à situação da flora epifítica vascular que ocorre nestas condições.

O presente trabalho aborda aspectos da florística e da ecologia dos epífitos vasculares que ocorrem sobre 60 figueiras, pertencentes a espécie Ficus organensis (Miq.) Miq., isoladas em áreas antropizadas, na planície costeira setentrional do Rio Grande do Sul, enfatizando a composição florística, as categorias ecológicas, as adaptações para resistir à seca e as formas de dispersão de diásporos das espécies encontradas.

\section{Material e métodos}

Área de estudo - a pesquisa foi realizada no norte da planície costeira do Rio Grande do Sul, no período de maio/1998 a julho/1999, em 
área localizada nos municípios de Terra de Areia e Capão da Canoa (29 32' a $29^{\circ} 39^{\prime} \mathrm{S}$ e $50^{\circ} 00^{\prime}$ a $50^{\circ} 05^{\prime}$ W, Fig. 1). O clima é do tipo Cfa de Koeppen (Nimer 1979), com temperaturas médias anuais em torno de $19{ }^{\circ} \mathrm{C}$. A pluviosidade na estação meteorológica mais próxima, localizada em Maquiné, a cerca de $30 \mathrm{~km}$ da área de estudo, fica em torno de $1.600 \mathrm{~mm}$ anuais (Waechter 1992; Sevegnani \& Baptista 1996). Os solos variam de cambissolos, na proximidade da encosta do planalto (Serra Geral), a areias quartzosas, na região mais próxima ao Oceano Atlântico (IBGE 1986). O relevo é plano a suavemente ondulado, com altitudes variando em torno de 20m (Ministério do Exército 1979). A vegetação local encontra-se bastante alterada pela ação antrópica, havendo alguns fragmentos de florestas arenosas, turfosas e pluviais.

Forófito - Ficus organensis (Miq.) Miq. pertence ao subgênero Urostigma, seção Americana (Carauta 1989), apresentando hábito hemiepifítico. As plântulas nascem sobre outras árvores e lançam raízes que atingem o solo, desenvolvem-se e terminam por estrangular a árvore hospedeira (Vásquez-Avila 1981; Daniels \& Lawton 1993; Hallé 1995; Putz et al. 1995; Swagel et al. 1997). A espécie distribui-se de Minas Gerais até o Rio Grande do Sul, onde assume grande importância fisionômica (Carauta 1989; Mello-Filho \& Neves 1989; Rambo 1994). Este fato, além da sua arquitetura, que favorece o desenvolvimento dos epífitos (Waechter 1992), motivou a escolha da espécie para a realização do presente trabalho

Procedimento amostral - foram amostrados apenas indivíduos arbóreos de Ficus organensis. Considerou-se como "figueiras isoladas" aquelas que não fazem parte do dossel de uma floresta. Assim, também foram incluídas figueiras que tenham uma outra árvore próxima (mesmo que as copas se toquem), ou que estejam agrupadas, formando um "parque". Como na região onde se localiza a área de estudo ocorrem florestas pluviais, arenosas, turfosas, senso
Waechter (1985), e a zona urbana da cidade de Terra de Areia, a seleção das árvores pesquisadas foi feita de modo a obter-se o mesmo número de árvores $(\mathrm{n}=15)$ próximas a cada um destes quatro ambientes. No total, foram amostradas 60 figueiras, com perímetro à altura do peito $\geq 30 \mathrm{~cm}$. As árvores foram escaladas e os epífitos vasculares registrados. Em algumas, devido ao grande porte, fez-se necessário o uso de equipamentos de alpinismo.

Identificação taxonômica - as espécies foram identificadas com o auxílio de monografias ou revisões taxonômicas de famílias ou gêneros que apresentam representantes na flora epifítica vascular regional e através de consultas a especialistas diversos e ao Herbário do Departamento de Botânica da UFRGS (ICN), onde foram depositadas as exsicatas relativas a este trabalho. Foram excluídas as espécies exóticas, plantadas e que não se reproduzem naturalmente (Dendrobium nobile Lindl.), e epífitos acidentais de ciclo anual (Solanum americanum Nill e Acnistus sp.).

Categorias ecológicas, adaptações ao estresse hídrico e formas de dispersão - a categoria ecológica dos epífitos foi definida de acordo com os critérios adotados por Waechter (1992), classificando-os como holoepífitos habituais (presentes principalmente em ambientes epidêndricos), facultativos (tanto ambientes epidêndricos como terrestres) ou acidentais (preferencialmente terrestres); e hemiepífitos (epífitos que têm conexão com o solo em alguma fase de sua vida). Neste último caso, foram considerados apenas aqueles encontrados na fase epifítica sobre as árvores amostradas. Os percentuais obtidos para estas categorias, bem como o número de espécies, foram comparados com outras estações amostradas. Foi registrada, para cada espécie de epífito vascular, a presença das seguintes adaptações morfológicas relacionadas com a resistência à seca (Waechter 1992; Benzing 1995): suculência foliar, suculência caulinar, 
velame radicular, tricomas foliares absorventes com a formação de cisternas para acúmulo de água, tricomas foliares absorventes sem a formação de cisternas, tricomas caulinares e poiquiloidria. Esta última adaptação, caracterizada pela capacidade da planta ressecar-se sem danificar seu conteúdo protoplasmático (Andrade \& Nobel 1997), foi a única adaptação fisiológica registrada por ser facilmente constatada a campo. A forma de dispersão de diásporos também foi abordada, distinguindo-se as seguintes categorias: anemocoria, que inclui diásporos diminutos (de dimensões próximas ou inferiores a $1 \mathrm{~mm}$ ) ou planadores (dotados de estruturas para planar); e zoocoria, incluindo diásporos carnosos ou viscosos.

\section{Resultados}

Foram encontradas 77 espécies de epífitos vasculares pertencentes a 33 gêneros e 10 famílias (Tab. 1), representando 30,8\% do número total de epífitos vasculares citados para a planície litorânea do Rio Grande do Sul. Orchidaceae (29 espécies) foi a família com a maior riqueza específica, porém com pouca importância fisionômica devido à baixa frequência de suas espécies. Apenas Brassavola tuberculata e Octomeria crassifolia foram mais conspícuas, formando densos aglomerados nas figueiras. Em Bromeliaceae (19 espécies), destacou-se o gênero Tillandsia, que foi encontrado em todas as figueiras amostradas, apresentando o maior número de espécies (9). Em termos fisionômicos, as Bromeliaceae que mais se destacaram foram Vriesea gigantea, devido ao grande porte dos indivíduos, e Tillandsia usneoides, facilmente identificável mesmo à distância. Entre as Cactaceae (9 espécies), que também apresentaram-se amplamente distribuídas, as espécies mais comuns foram Rhipsalis teres e Lepismium cruciforme. Nesta última espécie, os exemplares mais expostos ao sol apresentaram-se com uma coloração intensamente avermelhada, destacando-se nas árvores de copa menos densa. As demais famílias encontradas foram: Piperaceae (6 espécies), Polypodiaceae (5), Araceae (3), Gesneriaceae (3), Cecropiaceae (1), Dryopteridaceae (1) e Moraceae (1). Dentre as espécies pertencentes a estas famílias, Microgramma vacciniifolia e Microgramma squamulosa (Polypodiaceae) foram as que mais se destacaram, cobrindo extensas áreas da maioria dos forófitos. Na comparação com outros levantamentos (Tab. 2), o número de espécies de epífitos vasculares encontrado no presente trabalho (77) foi menor que o encontrado em Macaé de Cima (RJ) e em Torres (RS). Foi, ainda, igual ao encontrado na Ilha do Mel (PR) e maior que o encontrado em Arroio Bom Jardim, Emboaba e Taim (RS), Serra do Cipó (MG) e San Javier (Argentina).

Quanto à categoria ecológica, os holoepífitos habituais foram predominantes, com 69 espécies (89,61\%). Todas as espécies de Bromeliaceae, Orchidaceae e Polypodiaceae enquadraram-se nesta categoria ecológica, assim como a maioria das espécies de Cactaceae (Rhipsalis e Lepismium), Piperaceae (Peperomia), Araceae (Anthurium) e Gesneriaceae (Codonanthe). Os epífitos facultativos foram representados por três espécies (3,90\%): Sinningia douglasii (Gesneriaceae), encontrada em apenas uma árvore, em local abrigado nas reentrâncias do fuste; Rumohra adiantiformis (Dryopteridaceae), também encontrada em apenas um forófito e crescendo sob uma área protegida da copa da árvore; e Peperomia pereskiffolia (Piperaceae), que foi facilmente encontrada ocorrendo como terrestre, principalmente nas matas arenosas da região. Cereus hildmannianus e Opuntia arechavaletae (Cactaceae) foram as duas únicas espécies de epífitos acidentais encontrados (2,59\%). As árvores sobre as quais estavam estas cactáceas também ficavam próximas de matas arenosas, onde foi possível localizar exemplares de ambas com facilidade. Os hemiepífitos foram 
Tabela 1. Famílias e espécies de epífitos vasculares registrados nas figueiras (Ficus organensis) amostradas no norte da planície costeira do Rio Grande do Sul com a clasificação de sua respectiva categoria ecológica e tipo de diásporo.

\begin{tabular}{|c|c|c|}
\hline Família/Espécie & Cat.ecol. ${ }^{1}$ & Diásporo $^{2}$ \\
\hline \multicolumn{3}{|l|}{ ARACEAE } \\
\hline Anthurium scandens (Aubl.) Engl. & HAB & CAR \\
\hline A. gaudichaudianum Kunth & HAB & CAR \\
\hline Philodendron bipinnatifidum Schott ex Engl. & HEM & CAR \\
\hline \multicolumn{3}{|l|}{ BROMELIACEAE } \\
\hline Aechmea gamosepala Wittm. & HAB & CAR \\
\hline A. nudicaulis (L.) Griseb. & HAB & CAR \\
\hline A. recurvata (K1.) L. B. Smith & НАВ & CAR \\
\hline Billbergia zebrina (Herb.) Lindl. & HAB & CAR \\
\hline Tillandsia aeranthos (Loisel.) L. B. Smith & HAB & PLA \\
\hline T. gardneri Lindl. & HAB & PLA \\
\hline T. geminiflora Brongn. & HAB & PLA \\
\hline T. mallemontii Glaz. ex Mez & HAB & PLA \\
\hline T. recurvata $(\mathrm{L}.) \mathrm{L}$. & HAB & PLA \\
\hline T. stricta Soland. & HAB & PLA \\
\hline T. tenuifolia $\mathrm{L}$. & HAB & PLA \\
\hline T. tricholepis Backer & HAB & PLA \\
\hline T. usneoides (L.) L. & НАВ & PLA \\
\hline Vriesea friburgensis $\mathrm{Mez}$ & HAB & PLA \\
\hline V. gigantea Gaud. & HAB & PLA \\
\hline V.philippocoburgii Wawra & HAB & PLA \\
\hline V. procera (Mar. Ex Schult. f.) Wittm. & HAB & PLA \\
\hline V. rodigasiana $\mathrm{E}$. Morr. & HAB & PLA \\
\hline V. vagans L. B. Smith & HAB & PLA \\
\hline \multicolumn{3}{|l|}{ CACTACEAE } \\
\hline Cereus hildmannianus K. Schum. & ACI & CAR \\
\hline Lepismium cruciforme (Vell.) Miq. & HAB & CAR \\
\hline L. houlletianum (Lem.) Barthl. & HAB & CAR \\
\hline L. lumbricoides (Lem.) Barthl. & HAB & CAR \\
\hline L. warmingianum (K. Schum.) Barthl. & HAB & CAR \\
\hline Opuntia arechavaletae Speg. ex Arechav. & ACI & CAR \\
\hline Rhipsalis paradoxa Salm-Dyck & $\mathrm{HAB}$ & CAR \\
\hline R. pulvinigera Lindbg. & HAB & CAR \\
\hline R. teres (Vell.) Steud. & НAB & CAR \\
\hline \multicolumn{3}{|l|}{ CECROPIACEAE } \\
\hline Coussapoa microcarpa (Schott) Rizzini & HEM & CAR \\
\hline \multicolumn{3}{|l|}{ DRYOPTERIDACEAE } \\
\hline Rumohra adiantiformis (Forst.) Ching & FAC & DIM \\
\hline \multicolumn{3}{|l|}{ GESNERIACEAE } \\
\hline Codonanthe devosiana Lem. & HAB & CAR \\
\hline C. gracilis (Mart.) Hanst. & HAB & CAR \\
\hline Sinningia douglasii (Lindl.) Chaut. & FAC & DIM \\
\hline \multicolumn{3}{|l|}{ MORACEAE } \\
\hline Ficus organensis (Miq.) Miq. & HEM & CAR \\
\hline \multicolumn{3}{|l|}{ ORCHIDACEAE } \\
\hline Barbosella dusenii (A. Samp.) Schltr. & НAB & DIM \\
\hline
\end{tabular}


Tabela 1. (continuação)

Família/Espécie

Cat.ecol. $^{1}$

Diásporo $^{2}$

ORCHIDACEAE

Brassavola tuberculata Hook.

Campylocentrum aromaticum B. Rodr.

HAB

DIM

Cattleya intermedia Grah. Ex Hook.

HAB

DIM

Epidendrum pseudodifforme Schltr.

$\mathrm{HAB}$

DIM

E. latilabre Lindl.

$\mathrm{HAB}$

DIM

E. rigidum Jacq.

HAB

DIM

Isabelia pulchella (Krzl.) Sengh. \& Teusch.

HAB

DIM

Lanium avicula (Lindl.) Hoehne

HAB

DIM

Lankesterella ceracifolia (B. Rodr.) Mansf.

HAB

DIM

Maxillaria cogniauxiana Hoehne

HAB

DIM

Octomeria oxychela B. Rodr.

HAB

DIM

O. crassifolia Lindl.

HAB

DIM

O. gracilis Lodd.

Oncidium gravesianum Rolfe

$\mathrm{HAB}$

DIM

O. fimbriatum Lindl.

HAB

DIM

O. flexuosum Sims

HAB

DIM

HAB

DIM

O. micropogon Rchb. f.

$\mathrm{HAB}$

DIM

Pleurothallis hygrophila B. Rodr.

HAB

DIM

P. linearifolia Cogn.

HAB

DIM

P. aphtosa Lindl.

P. cf. marginalis Rchb. f.

HAB

DIM

HAB

DIM

P. exarticulata B. Rodr.

$\mathrm{HAB}$

DIM

P. glumacea Lindl.

$\mathrm{HAB}$

DIM

P. iguapensis Schltr.

$\mathrm{HAB}$

DIM

P. sarracenia Luer

Polystachya estrellensis Rchb. f.

$\mathrm{HAB}$

DIM

HAB

DIM

Prosthechea vespa (Vell.) W.E. Higgins

$\mathrm{HAB}$

DIM

HAB

DIM

Sophronitis purpurata (Lindl.) van den Berg \& Chase

HAB

DIM

\section{PIPERACEAE}

Peperomia alata Ruiz \& Pavon

P. catharinae Miq.

P. pereskiifolia (Jacq.) HBK.

P. glabella (Sw.) A. Dietr.

P. rotundifolia (L.) HBK.

P. tetraphylla (Forst.) Hook. \& Arn.

$\begin{array}{ll}\text { HAB } & \text { VIS } \\ \text { HAB } & \text { VIS } \\ \text { FAC } & \text { VIS } \\ \text { HAB } & \text { VIS } \\ \text { HAB } & \text { VIS } \\ \text { HAB } & \text { VIS }\end{array}$

\section{POLYPODIACEAE}

Microgramma squamulosa (Kaulf.) Sota

HAB

DIM

M. vacciniifolia (Langsd. \& Fisch) Copel.

HAB

DIM

Polypodium hirsutissimum Raddi

HAB

DIM

P. pleopeltifolium Raddi

HAB

DIM

P. squalidum Vell.

HAB

DIM

1 Categorias ecológicas: HAB = holoepífitos habituais; ACI = holoepífitos acidentais; FAC = holoepífitos facultativos; HEM = hemiepífitos. ${ }^{2}$ Tipos de diásporos: DIM = diásporos diminutos; PLA $=$ diásporos planadores; CAR $=$ diásporos carnosos; VIS = diásporos viscosos. 
representados por três espécies $(3,90 \%)$ : Coussapoa microcarpa (Cecropiaceae), Ficus organensis (Moraceae) e Philodendron bipinnatifidum (Araceae). As duas primeiras apresentaram hábito estrangulador. $C$. microcarpa foi representada por exemplares vigorosos desenvolvendo-se, preferencialmente, no alto do fuste dos seus hospedeiros. $F$. organensis foi encontrada como epífita em três figueiras da m esma espécie. Em pelo menos um caso, a árvore hospedeira apresentava-se debilitada pelo desenvolvimento da figueira mais jovem. A terceira espécie, P. bipinnatifidum (Araceae), ocorre como terrícola nas proximidades das figueiras sobre as quais foi encontrada. Estas proporções entre as categorias ecológicas foram semelhantes às encontradas em outros levantamentos da sinúsia epifítica (Tab. 2).

Freqüentemente, as espécies epifíticas apresentaram mais de uma adaptação morfológica para resistir à seca (Tab. 3). Apenas em Ficus organensis e Coussapoa microcarpa não se identificou adaptação morfológica (2,59\%). A suculência foliar ocorreu nas duas espécies de Codonanthe (Gesneriaceae); em Barbosella dusenii, Brassavola tuberculata,
Campylocentrum aromaticum, Cattleya intermedia, Lankesterella ceracifolia, Sophronitis purpurata, Epidendrum (três espécies), Octomeria (três espécies) e nas oito espécies de Pleurothallis (Orchidaceae); e nas seis espécies de Peperomia (Piperaceae), totalizando $37,66 \%$ das espécies de epífitos vasculares. A suculência caulinar foi encontrada nas nove espécies de Cactaceae, nas duas de Microgramma (Polypodiaceae); em Rumohra adiantiformis (Dryopteridaceae); em Campylocentrum aromaticum, Cattleya intermedia, Prosthechea vespa, Isabelia pulchella, S. purpurata, Lanium avicula, Maxillaria cogniauxiana, Polystachya estrellensis e nas quatro espécies de Oncidium (Orchidaceae); e em Sinningia douglasii (Gesneriaceae), totalizando 32,46\% das espécies. $\mathrm{O}$ velame, tecido radical especializado para absorção e retenção de água, foi encontrado em Orchidaceae e Araceae (41,55\% das espécies). A presença de tricomas foliares absorventes sem a formação de cisternas foi constatada em Tillandsia (Bromeliaceae, $11,68 \%$ das espécies), permitindo a captação de água diretamente da atmosfera. Nos demais gêneros de Bromeliaceae (Aechmea, Billbergia

Tabela 2. Número de espécies e proporções das categorias ecológicas das espécies epifíticas vasculares encontradas sobre figueiras isoladas e em levantamentos realizados em áreas florestais do Rio Grande do Sul e de outras localidades.

\begin{tabular}{|c|c|c|c|c|c|}
\hline \multirow[b]{2}{*}{ Local $^{1}$} & \multirow{2}{*}{$\begin{array}{l}\text { Número de } \\
\text { Espécies }\end{array}$} & \multicolumn{4}{|c|}{ Categorias ecológicas $(\%)^{2}$} \\
\hline & & HAB & ACI & FAC & HEM \\
\hline Macaé de Cima $(\mathrm{RJ}) \dagger$ & 311 & 80,9 & 0 & 13,9 & 5,2 \\
\hline Litoral do Rio Grande do Sul* & 250 & 86,8 & 5,2 & 2,6 & 3,6 \\
\hline Faxinal $(\mathrm{RS})^{3 * * * *}$ & 120 & 94,2 & 0 & 4,2 & 1,6 \\
\hline F. organensis isoladas (este estudo) & 77 & 89,6 & 2,6 & 3,9 & 3,9 \\
\hline Ilha do Mel (PR)** & 77 & 70,0 & 5,0 & 18,0 & 6,0 \\
\hline Emboaba (RS) $* * *$ & 53 & 79,2 & 7,6 & 7,6 & 5,6 \\
\hline Taim $(\mathrm{RS}) *$ & 24 & 79,2 & 4,2 & 16,6 & 0 \\
\hline Serra do Cipó (MG) $\dagger \dagger \dagger$ & 29 & 89,6 & 3,5 & 6,9 & 0 \\
\hline Arroio Bom Jardim (RS) $* * * * *$ & 17 & 100,0 & 0 & 0 & 0 \\
\hline San Javier (Argentina) $\dagger \dagger$ & 17 & 88,2 & 11,8 & 0 & 0 \\
\hline
\end{tabular}

${ }^{1}$ Local: $*=$ Waechter $(1992) ; * *=$ Kersten \& Silva $(2001) ; * * *=$ Waechter $(1998 b) ; * * * *=$ Waechter $(1986) ; * * * * *=$ Aguiar et al. (1981); $\dagger=$ Fontoura et al. (1997); $\dagger \dagger=$ Roldán (1995), $\dagger \dagger \dagger=$ Meguro et al. (1996). ${ }^{2}$ Acrônimos das categorias ecológicas conforme Tab. 1. ${ }^{3}$ Exclusive hemiepífitos primários (estranguladores). 
Tabela 3. Principais adaptações de resistência ou evitamento da seca encontradas em epífitos vasculares que ocorrem nas figueiras isoladas no norte da planície costeira do Rio Grande do Sul. N = número total de espécies por tipo de adaptação.

\begin{tabular}{lcl}
\hline Adaptação & $\mathrm{N}$ & Distribuição por família $\left(\mathrm{n}^{1}\right)$ \\
\hline Velame & 32 & Araceae (3), Orchidaceae (29) \\
Tricomas foliares & 19 & Bromeliaceae (19) \\
Tricomas caulinares & 2 & Polypodiaceae (2) \\
Cisternas ou tanques & 10 & Bromeliaceae (10) \\
Suculência caulinar & 25 & Cactaceae (9), Dryopteridaceae (1), \\
& & Gesneriaceae (1), Orchidaceae (12), \\
Suculência foliar & 29 & Polypodiaceae (2) \\
Poiquiloidria & 3 & Dryopteridaceae (1), Gesneriaceae (2), \\
& & Polypodiaceae (3)
\end{tabular}

${ }^{1} \mathrm{n}=$ Número de espécies por família.

e Vriesea) ocorreram, além dos tricomas foliares absorventes, cisternas para acumulação de água $(12,99 \%$ dos epífitos). Entre as Polypodiaceae, Microgramma apresentou o caule crasso e reptante recoberto por tricomas caulinares $(2,60 \%)$, enquanto o gênero Polypodium apresentou poiquiloidria (3,89\%).

A anemocoria foi a forma de dispersão de diásporos de $66,24 \%$ das espécies (51), das quais 36 apresentaram diásporos diminutos e 15 diásporos planadores. A zoocoria esteve presente em $33,76 \%$ espécies (26), das quais 20 apresentaram diásporos carnosos. Os diásporos viscosos ocorreram em todas as seis espécies de Peperomia.

\section{Discussão}

O grande número de espécies encontrado no presente estudo demonstra que a conservação de árvores nativas isoladas, em ambientes antropizados, pode garantir a manutenção de parcela significativa da flora epifítica regional, como ressaltado por Willians-Linera et al. (1995) e Guevara et al. (1998). No entanto, é interessante observar que, no caso de Ficus organensis, a maioria das árvores aparentemente nasceu ou foi plantada fora do ambiente florestal (C. N. Gonçalves, dados não publicados), ao contrário do que acontece nos trabalhos referidos anteriormente, onde as árvores isoladas normalmente são relictos de florestas que ocorriam nas áreas estudadas (Willians-Linera et al. 1995; Guevara et al. 1998). Desta forma, as figueiras são mais importantes por representarem um ambiente propício para a colonização de epífitos vasculares nas áreas antropizadas do que como mantenedoras de remanescentes da flora epifítica florestal.

Orchidaceae é constantemente citada como a família botânica mais diversa em levantamentos da flora epifítica vascular (Waechter 1986, 1998b; Kelly et al. 1994; Fontoura et al. 1997; Rudolph et al. 1998), o que também se verificou no presente estudo. O sucesso desta família é creditado aos numerosos e diminutos diásporos, permitindo dispersão bem sucedida a médias e longas distâncias (Pabst \& Dungs 1977; Nunes \& Waechter 1998; Waechter 1998a), e a outras adaptações e especializações, como a presença de velame e de pseudobulbos (órgãos caulinares suculentos especializados para a retenção de água), relações com polinizadores e a relação simbiótica com fungos micorrízicos. Tais adaptações, entretanto, podem torná-las exigentes quanto aos nichos ecológicos que ocupam e restringir a sua ocorrência (Pijl \& Dodson 1969; Zimmerman \& Olmsted 1992; 
Migenis \& Ackerman 1993; Wolf 1994; Freiberg 1996; Tremblay et al. 1998; Singer \& Cocucci 1999). Provavelmente devido a estas características, a maioria das espécies desta família encontradas no presente estudo apresentou ocorrência “pontual", ou seja, restrita a poucas figueiras (Gonçalves 2000).

A grande representatividade de Bromeliaceae, no presente trabalho, não apenas quanto à diversidade, mas também pela importância fisionômica, está de acordo com os dados apresentados por Reitz (1983), que cita a ocorrência de mais de 3.000 exemplares de Bromeliaceae em um único indivíduo de F. organensis na floresta atlântica, em Santa Catarina. A riqueza desta família nas figueiras isoladas pode ser creditada a diversos fatores, porém um dos mais importantes, possivelmente, é a grande intensidade luminosa a qual a flora epifítica está submetida nas figueiras isoladas (Sillett et al. 1995), favorecendo táxons heliófilos, como é o caso das espécies de Tillandsia e Vriesea, que compõem 78,95\% do total de espécies desta família nas árvores analisadas.

A forte participação dos gêneros Lepismium e Rhipsalis (Cactaceae) e Microgramma (Polypodiaceae) na fisionomia da flora epifítica regional é ressaltada por Waechter (1992). Microgramma vacciniifolia é citada constantemente como a espécie que apresenta os maiores valores de importância entre as espécies epifíticas no litoral do Rio Grande do Sul, tanto por sua freqüência elevada (Waechter 1992, 1998b) como por sua grande cobertura nos forófitos (Gonçalves 2000).

A participação das categorias ecológicas de epífitos vasculares encontrados sobre F. organensis, em valores percentuais, é muito semelhante às encontradas em outros levantamentos (Waechter 1986, 1992, 1998b; Roldán 1995; Fontoura et al. 1997; Dittrich et al. 1999; Kersten \& Silva 2001). O forte predomínio de holoepífitos habituais reforça importância de $F$. organensis isoladas, pois estas espécies só podem se desenvolver no ambiente epifítico. Dentre as demais categorias ecológicas, a classificação das espécies em acidentais ou facultativas muitas vezes depende da circunstância. Apenas Peperomia pereskiifolia, entre as espécies classificadas nesta categoria, mostra-se claramente facultativa quanto à ocorrência como epífita ou como terrícola (Waechter 1998b). Assim, Sinningia douglasii, caracterizada por apresentar um "tubérculo", segundo a terminologia de Toursarkissian (1969), do qual partem seus ramos, foi considerada como facultativa devido a sua ocorrência nos afloramentos rochosos da Serra Geral (Waechter 1992). Rumohra adiantiformis é considerada, algumas vezes, como facultativa (Waechter 1992) e, em outras ocasiões, como epífita acidental (Waechter 1998b). A opção pela primeira categoria, neste trabalho, deveu-se a freqüente presença desta espécie tanto no solo como em árvores nas matas da área de estudo. Por outro lado, os fustes e os ramos de F. organensis favorecem o acúmulo de matéria orgânica, o que ocasiona a formação de húmus e solos epifíticos. Nesta circunstância, espécies de plantas tipicamente terrestres podem germinar e se desenvolver, como os dois epífitos acidentais encontrados, Oputina arechavaletae e Cereus hildmannianus. Waechter (1992) destaca a presença freqüente destas duas espécies sobre $F$. organensis no litoral do Rio Grande do Sul. Quanto aos hemiepífitos, que têm recebido atenção cada vez maior devido à sua significativa participação na físionomia de florestas tropicais (Daniels \& Lawton 1993; Putz et al. 1995; Willians-Linera \& Lawton 1995), são pouco representados nas figueiras analisadas.

A predominância da dispersão anemocórica no litoral sul-rio-grandense já havia sido apontada por Waechter (1992), mas o percentual de espécies com esta estratégia de dispersão é menor nas figueiras $(66,24 \%$, contra $80,4 \%$ para todo o litoral). Em áreas florestais próximas da 
área onde se realizou o presente estudo, o percentual de espécies anemocóricas encontrado também foi maior, totalizando $80,83 \%$ das espécies epifíticas na mata do Faxinal (Waechter 1986) e 73,58\% em Emboaba (Waechter 1998b). Um dos principais motivos é a menor representatividade, nas árvores amostradas, de táxons da família Orchidaceae e de Pteridófitas - Dryopteridaceae (Rumohra adiantiformis) e Polypodiaceae (Polypodium e Microgramma), que representam $8,75 \%$ das espécies. Entre os táxons com dispersão por zoocoria, os diásporos viscosos de Peperomia são, provavelmente, epizoocóricos (Pijl 1969). Os diásporos carnosos podem ser adaptados para a dispersão por aves (ornitocoria) ou morcegos (quiropterocoria). As espécies ornitocóricas têm as cores dos diásporos vivas (por exemplo, Aechmea nudicaulis, Rhipsalis pulvinigera e Lepismium cruciforme), contrastando com o ambiente, e sem odor (Bregman 1988). Nas espécies quiropterocóricas, a cor costuma ser pouco destacada (branco ou verde), com odores que facilitem sua localização (por exemplo, Anthurium gaudichaudianum e Rhipsalis teres).

\section{Agradecimentos}

Aos funcionários do Herbário ICN, aos professores do PPG Botânica da UFRGS e aos revisores, cujas sugestões muito contribuíram para a melhoria do original. $\mathrm{O}$ primeiro autor agradece a Cristiane F. de Azevêdo-Gonçalves pelo auxílio nos trabalhos de campo e ao CNPQ, pela bolsa concedida.

\section{Referências bibliográficas}

Aguiar, L. W.; Citadini-Zanette, V.; Martau, L. \& Backes, A. 1981. Composição florística de epífitos vasculares numa área localizada nos municípios de Montenegro e Triunfo, Rio Grande do Sul, Brasil. Iheringia, Série Botânica 28: 55-93.

Andrade; J. L. \& Nobel, P. S. 1997. Microhabitats and water relations of epiphytic cacti and ferns in a lowland neotropical forest. Biotropica 29(3): 261-270.
Atzingen, N. von; Cardoso, A. L. R. \& Ilkiu-Borges, A. L. 1996. Flora orquidológica da Serra das Andorinhas, São Geraldo do Araguaia - PA. Boletim do Museu Paraense Emílio Goeldi, Série Botânica 12(1): 59-74.

Benzing, D. H. 1995. Vascular epiphytes pp. 225-254. In M. D. Lowman \& N. M. Nadkarni (eds.). Forest canopies. $1^{\text {st }}$ ed. Academic Press, San Diego.

Braga, M. M. N. \& Braga, P. I. S. 1975. Estudos sobre a vegetação das campinas amazônicas IV. Ocorrência de algumas epífitas na campina e campinarana. Acta Amazonica 5(3): 254-260.

Bregman, R. 1988. Forms of seed dispersal in Cactaceae. Acta Botanica Neerlandesa 37(3): 395-402.

Brown, A. D. 1990. Epyphytism in the montane forests of El Rey National Park in Argentina Floristic composicion and distribution pattern. Revista Biología Tropical 38(2A): 155-166.

Carauta, J. P. P. 1989. Ficus (Moraceae) no Brasil: conservação e taxonomia. Albertoa 2: 1-365.

Catling, P. M.; Brownnell, V. R. \& Lekovitch, L. P. 1986 Epiphytic orchids in a Belizean grapefruit orchard: distribution, colonization and association. Lindleyana 1: 194-202.

Coxson, D. S. \& Nadkarni, N. M. 1995. Ecological roles of epiphytes in nutritent cycles of forest ecossystems pp. 27-44. In M. D. Lowman \& N. M. Nadkarni (eds.). Forest canopies. $1^{\text {st }}$ ed. Academic Press, San Diego.

Daniels, J. D. \& Lawton, R. O. 1993. A natural history of strangling by Ficus crassiuscula in a Costa Rican lower montane rain forest. Selbyana 14(1): 59-63.

Dislich, R. \& Mantovani, W. 1998. A flora de epífitas da reserva da Cidade Universitária "Armando de Salles Oliveira" (São Paulo, Brasil). Boletim de Botânica da Universidade de São Paulo 17: 61-84.

Dittrich, V. A. O., Kozera, C. \& Menezes-Silva, S. 1999. Levantamento florístico dos epífitos vasculares do Parque Barigüi, Curitiba, Paraná, Brasil. Iheringia, Série Botânica 52: 11-21.

Fontoura, T.; Sylvestre, L. S.; Vaz, A. M. S. \& Vieira, C. M. 1997. Epífitas vasculares, hemiepífitas e hemiparasitas da Reserva Ecológica de Macaé de Cima pp.89-101. In H. C. Lima \& R. R. GuedesBruni. Serra de Macaé de Cima: diversidade florística e conservação em Mata Atlântica. Jardim Botânico do Rio de Janeiro, Rio de Janeiro.

Freiberg, M. 1996. Spatial distribution of vascular epiphytes on three emergent canopy trees in French Guiana. Biotropica 28(3): 345-355. 
Galeano, G.; Suarez, S. \& Balslev, H. 1998. Vascular plant species count in a wet forest in the Choco area on the Pacific coast of Colombia. Biodiversity and Conservation 7(12): 1563-1575.

Gonçalves, C. N. 2000. Epifitismo vascular sobre figueiras isoladas no norte da Planície Costeira do Estado do Rio Grande do Sul. Dissertação de Mestrado. Universidade Federal do Rio Grande do Sul. Porto Alegre.

Gottsberger, G. \& Morawetz, W. 1993. Development and distribution of the epiphytic flora in na Amazonian savanna in Brazil. Flora 1: 145-151.

Guevara, L.; Laborde, J. \& Sánchez, G. 1998. Are isolated remnant trees in pastures a fragmented canopy? Selbyana 19(1): 34-43.

Hallé, F. 1995. Canopy architecture in tropical trees: a pictorial approach pp. 27-44. In M. D. Lowman $\&$ N. M. Nadkarni (eds.). Forest canopies. $1^{\text {st }}$ ed. Academic Press, San Diego.

Heatwole, H. 1990. Distribution of epiphytes on trunks of the arborescent fern, Blechnum palmiforme, at Gough island, South Atlantic. Selbyana 14(1): 46-58.

Hietz, P. 1997. Population dynamics of epiphytes in a Mexican humid montane forest. Journal of Ecology 85(6): 767-775.

Hietz, P. \& Hietz-Seifert, U. 1995. Composition and ecology of vascular epiphyte communities along an altitudinal gradient in central Veracruz, Mexico. Journal of Vegetation Science 6(4): 487-498.

IBGE. 1986. Levantamento de recursos naturais. v. 33. Instituto Brasileiro de Geografia e Estatística, Rio de Janeiro, Brasil.

Ingram, S. W.; Ferrell-Ingram, K. \& Nadkarni, N. M. 1996. Floristic composition of vascular epiphytes in a neotropical cloud forest, Monteverde, Costa Rica. Selbyana 17(1): 88-103.

Jarman, S. J. \& Kantvilas, G. 1995. Epiphytes on an old huon pine tree (Lagarostrobos franklinii) in Tasmanian rain-forest. New Zealand Journal of Botany 33(1): 65-78.

Kelly, D. L.; Tanner, E. V. J.; Lughadha, E. M. N. \& Kapos, V. 1994. Floristics and biogeography of a rain-forest in the Venezuelan Andes. Journal of Biogeography 21(4): 421-440.

Kersten, R. A. \& Menezes-Silva, S. 2001. Composição florística e estrutura do componente epifítico vascular em floresta de planície litorânea na Ilha do Mel, Paraná, Brasil. Revista Brasileira de Botânica 24(2): 213-226.

Labiak, P. H. \& Prado, J. 1998. Pteridófitas epífiticas da Reserva Volta Velha, Itapoá - Santa Catarina, Brasil. Boletim do Instituto de Botânica 11: 1-79.
Medeiros, A. C.; Loope, L. L. \& Anderson, S. J. 1993. Differencial colonization by epiphytes on native (Cibotium spp.) and alien (Cyathea cooperi) ferns in a Hawaiian rain forest. Selbyana 14(1): 71-74.

Mello Filho, L. E. \& Neves, L. J. 1989. Anatomia foliar de Ficus organensis (Miq.) Miq. Bradea 5(15): 156-165.

Migenis, L. E. \& Ackerman, J. D. 1993. Orchidphorophyte relationships in a forest watershed in Puerto Rico. Journal of Tropical Ecology 9: 231-240.

Ministério do Exército. 1979. Maquiné. Região Sul do Brasil - 1:50.000, Folha SH.22-X-C-V-2 MI-297/2. (Carta cartográfica).

Moguel, P. \& Toledo, V. M. 1999. Biodiversity conservation in traditional coffee systems of Mexico. Conservation Biology 13(1): 11-21.

Nadarajah, P. \& Nawawi, A. 1993. Mycorrhizal status of epiphytes in Malaysian oil palm plantations. Mycorrhyza 4(1): 21-25.

Nieder, J.; Engwald, S. \& Barthlott, W. 1999. Patterns of neotropical epiphyte diversity. Selbyana 20(1): 66-75.

Nimer, E. 1979. Climatologia do Brasil. IBGESUPREN, Rio de Janeiro, Brasil. 421 p.

Nunes, V. F. \& Waechter, J. L. 1998. Florística e aspectos fitogeográficos de Orchidaceae epifíticas de um morro granítico subtropical. Pesquisas 48: 127-162.

Pabst, G. F. J. \& Dungs, F. 1977. Orchidaceae Brasilienses. Hildesheim, Brücke, Alemanha. v. 2. $418 \mathrm{p}$.

Pijl, L. V. D. 1969. Principles of dispersal in higher plants. Springer. Berlin. $153 \mathrm{p}$.

Pijl, L. V. D, \& Dodson, C. H. 1969. Orchid flowers their pollination and evolution. University of Miami Press. Coral Gables. 214 p.

Putz, F. E.; Romano, G. B. \& Holbrook, N. M. 1995. Comparative phenology of epiphytic and tree phase strangle fig in a Venezuelan palm savannah. Biotropica 27(2) 183-189.

Rambo, B. 1994. A fisionomia do Rio Grande do Sul. $3^{\mathrm{a}}$ ed. Editora da Universidade do Vale do Rio dos Sinos, São Leopoldo, Brasil. 473p.

Reitz, R. 1983. Bromeliáceas e a malária-bromélia endêmica. Flora Ilustrada Catarinense (brom): $1-559$.

Ribeiro, J. E. L. D.; Garcia, J. P. M. \& Monteiro, R. 1994. Distribution of the orchid species in the coastal plains of the Fazenda Beach, municipality of Ubatuba, state of São Paulo, Brazil. Arquivos de Biologia e Tecnologia 37(3): 515-526. 
Roldán, A. I. 1995. Estructura de sitios disturbados y no disturbados de las selvas montanas de la sierra de San Javier (Tucumán, Argentina). Lilloa 38(2): 133-156.

Rudolph, D.; Rauer, G.; Neider, J. \& Barthlott, W. 1998. Distributional patterns of epiphytes in the canopy and phorophyte characteristics in a western Andean rain forest in Ecuador. Selbyana 19(1): 27-33.

Sevegnani, L. \& Baptista, L. R. M. 1996. Composição florística de um floresta secundária, no âmbito da Floresta Atlântica, Maquiné, RS. Sellowia 25-48: 47-71.

Sillett, S.; Gradstein, S. R. \& Griffin III, D. 1995. Bryophyte diversity of Ficus tree crowns from cloud forest and pasture in Costa Rica. The Bryologist 98:(2) 251-260.

Silva, M. F. F.; Silva, J. B. F. \& Feiler, J. M. 1999. Orchidáceas do Estado do Maranhão, Brasil. Acta Amazonica 12(3): 381-394.

Singer, R. B. \& Cocucci, A. A. 1999. Pollination mechanism in southern Brazilian orchids which are exclusively or mainly pollinated by halictid bees. Plant Systematics and Evolution 217(1-2): 101-117.

Swagel, E. N.; Bernard, A. van H. \& Ellmore, G. S. 1997. Substrate water potential constraints on germination of the strangler fig Ficus aurea (Moraceae). American Journal of Botany 84(5) 716-722.

Toursarkissian, M. 1969. Las Gesneriáceas argentinas. Darwiniana 15(1-2): 25-64.

Tremblay, R. L.; Zimmerman, J. K.; Lebron, L.; Bayman, P.; Sastre, I.; Axelrod, F. \& Alers-Garcia, J. 1998. Host specificity and low reproductive success in the rare endemic Puerto Rican orchid Lepanthes caritensis. Biological Conservation 85(3): 297-304.
Vásquez-Avila, M. D. 1981. El género Ficus (Moraceae) en la República Argentina. Darwiniana 23(2-4): 605-636.

Waechter, J. L. 1985. Aspectos ecológicos da vegetação de restinga do Rio Grande do Sul, Brasil. Comunicações do Museu de Ciências PUCRS, Série Botânica 33: 49-68.

Waechter, J. L. 1986. Epífitos vasculares da mata paludosa do Faxinal, Torres, Rio Grande do Sul, Brasil. Iheringia, Série Botânica 34: 39-49.

Waechter, J. L. 1992. O epifitismo vascular na Planície Costeira do Rio Grande do Sul. Tese de Doutorado. Universidade Federal de São Carlos. São Carlos.

Waechter, J. L. 1998a. Epiphytic orchids in eastern subtropical South America Pp. 332-341. In Proceedings of the $15^{\text {th }}$ World Orchid Conference, Rio de Janeiro. Naturalia Publications, Turriers.

Waechter, J. L. 1998b. Epifitismo vascular em uma floresta de restinga do Brasil subtropical. Revista Ciência e Natura 20: 43-66.

Willians-Linera, G. \& Lawton, R. O. 1995. The ecology of hemiepiphytes in forest canopies Pp. 255-283. In M. D. Lowman. \& N. M. Nadkarni (eds.). Forest canopies. $1^{\text {st }}$ ed. Academic Press, San Diego.

Willians-Linera, G.; Sosa, V. \& Platas, T. 1995. The fate of epiphytic orchids after fragmentation of a Mexican cloud forest. Selbyana 16(1): 36-40.

Wolf, J. H. D. 1994. Factors controlling the distribution of vascular and non vascular epiphytes in the northern Andes. Vegetatio 112(1): 15-28.

Yeaton, R. I. \& Gladstone, D. E. 1982. The pattern of colonization of epiphytes on calabash trees (Crescentia alata) in Guanacaste province, Costa Rica. Biotropica 14(2): 137-140.

Zimmerman, J. K. \& Olmsted, I. C. 1992. Host tree utilization by vascular epiphytes in a seasonally inundated forest (tintal) in Mexico. Biotropica 24(3): 402-407. 